\title{
Analysis of basal physical fitness and lumbar muscle function according to indoor horse riding exercise
}

\author{
Chang Ho Yu ${ }^{\mathrm{a}}$, Chul Un Hong ${ }^{\mathrm{b}}$, Seung Rok Kang ${ }^{\mathrm{b}}$ and Tae Kyu Kwon ${ }^{\mathrm{a},{ }^{*}}$ \\ ${ }^{a}$ Division of Biomedical Engineering, Chonbuk National University, Deokjin-Dong 1-Ga, Jeonju, \\ Jeonbuk 561-756, South Korea \\ ${ }^{b}$ Department of Healthcare Engineering, Chonbuk National University, Deokjin-Dong 1-Ga, Jeonju, \\ Jeonbuk 561-756, South Korea
}

\begin{abstract}
The aim of this study is to verify the effect of indoor horse riding exercise on basal physical exercise and lumbar muscular function. The subjects included were 20 healthy females, who participated in the horse riding exercise using SRider (Rider Co. \& ChonbuK National Univ, Korea) for 30 minutes per day, 3 days per week, over a period of 8 weeks. The subjects were divided into 4 groups as follows, with 10 subjects in each group: Postural Balance Exercise mode (PBE), Abdomen Exercise mode (ADE), Whole body Exercise mode (WBE), and Multiple Exercise (MTE). Isokinetic muscular function test was performed before and after the horse riding exercise, to assess the effect of horse riding on basal physical exercise and lumbar muscular function. The test result on basal physical exercise and isokinetic muscular function showed improvements with variable degree in the back muscle strength, maximum joint torque, total work, and muscular acceleration time. The result signifies that the horse riding is an antagonistic exercise mainly performed on waist and abdomen area, and the machine induces persistent muscle contraction and causes myotonic induction enhancing the muscle strength. Indoor horse riding exercise proved its effectiveness for senior or the disabled people who need muscle exercises but have difficulties performing outdoor activities.
\end{abstract}

Keywords: Horse riding, exercise effect, exercise instrument for indoor

\section{Introduction}

In modern society, the growth of economy and development of technology have enabled us to enjoy the convenient and abundant life. At the same time, such life style raises health issues and lack of exercise can lead to modern diseases such as chronic adult diseases [1]. Hence, there have been popular interests in the importance of working out, and exercises such as jogging, cycling, and stepping are performed using equipment such as tramp, running machine, stationary bicycle, or stepper. These are convenient indoor exercises using the whole body. However, many people stop using the exercise equipment after a short period of time due to boredom. The horse riding exercise has been gaining increasing attention as a solution for overcoming the boredom while exercising. A newer version of this

\footnotetext{
${ }^{*}$ Corresponding author: Tae Kyu Kwon, Division of Biomedical Engineering, Chonbuk National University, Deokjin-Dong 1-Ga, Jeonju, Jeonbuk 561-756, South Korea. Tel.: +82-63-270-4066; Fax: +82-63-270-2247; E-mail: kwon10@jbnu.ac.kr.
} 
equipment developed higher effectiveness [2,3]. This new equipment provides great exercise effects with its benefits of indoor use for seniors, disabled persons, and others who have difficulty in performing outdoor activities [4-8].

The horse riding exercise has been known as total-body workout with an outstanding exercise effect as well as excellent rehabilitation effect [9]. The core of horse riding exercise is a repetition of maintaining stance by matching the horse movement, and this is effective for enhancing postural balancing ability, muscular strength, and flexibility [10]. This 3D movement of horse has been known to be effective in promoting blood circulation and recovering deteriorated functions by activating muscles and joints through stimulating the core muscles that are not commonly used even by horse riders [11]. Horse riding produces an exercise effect similar to general low-strength exercises that are ideal for the aged people with pain in the knee or waist [12]. In addition, the effect of horse riding exercise has been proved through the studies defining the positive correlations between the horse riding exercise and the following factors: muscular endurance, agility, coordination, flexibility, balance, aerobic and anaerobic capacity $[13,14]$.

The horse riding has attracted great attention as a whole body exercise with many beneficial effects; and despite the high demand, it has not been popularized in Korea due to the limits of availability, location, and cost. This gap between the increasing demands and limited offers was covered by developing indoor horse riding machine. However, there have not been any researches on the exercise effect of the indoor horse riding equipment that are manufactured in Korea. Moreover, simulator horse riding equipment based on virtual reality technology emerged, but scientific verification will still be needed to recognize these as healthy exercise equipment.

Thus, this research was conducted to verify the effect of indoor horse riding on basal physical exercise and lumbar muscular function. A total of 20 females were included in this study, who exercised on the horse riding equipment that is already in the market manufactured by Korean technology.

\section{Experimental methods}

\subsection{Subjects}

The forty subjects, who had no horse riding exercise experience and no medical history or drug treatment, participated in this study. The subjects were divided into 4 groups as follows: Postural Balance Exercise mode (PBE ; age : 18.6yr , height : $161 \mathrm{~cm}$, weight : $53.6 \mathrm{~kg}$ ), Abdomen Exercise mode (ADE ; age : 19.7yr , height : $160.4 \mathrm{~cm}$, weight : $51.8 \mathrm{~kg}$ ), Whole body Exercise mode (WBE ; age : $19.5 \mathrm{yr}$, height : $159.3 \mathrm{~cm}$, weight : 49.9kg), and Multiple Exercise mode (MTE ; age : 19.2yr , height : $159.3 \mathrm{~cm}$, weight : $55.6 \mathrm{~kg}$ ). Each group consisted of ten subjects. All subjects were provided with explanation of the purpose and risks of experiment, and the deviation that can be caused by different heights and weights of the subjects were minimized to reduce errors.

\subsection{Experiment equipment}

SRider (Rider Co. \& Chonbuk National Univ, Korea) was used for the experiment in this study, which is an indoor horse riding exercise instrument commonly distributed in the local markets. As shown on Figure 1, its shape simulates a horseback and the exercise strength can be controlled by adjusting the speed and range of saddle movement. 

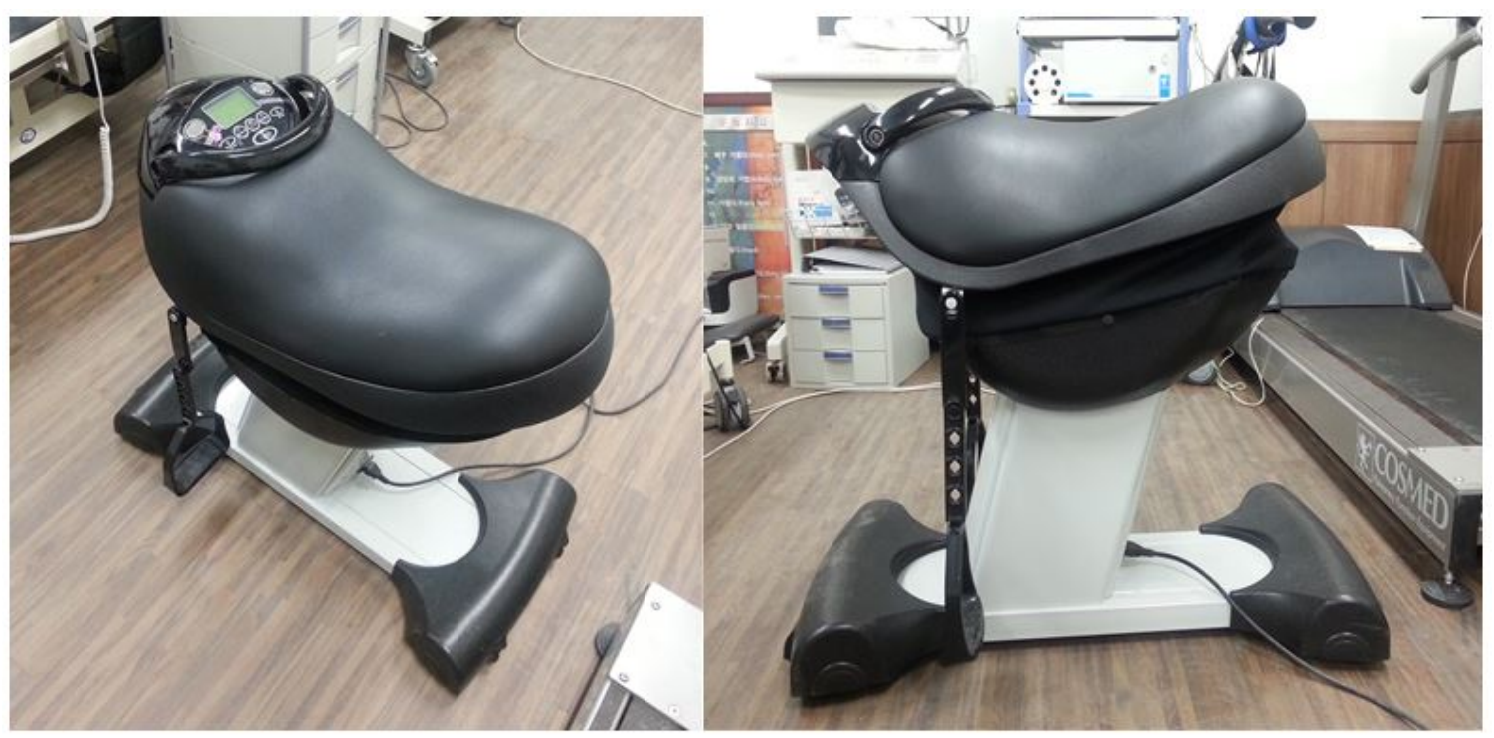

Fig. 1. (a) SRider is a horse riding exercise instrument (Rider Co. \& Chonbuk National Univ Korea).

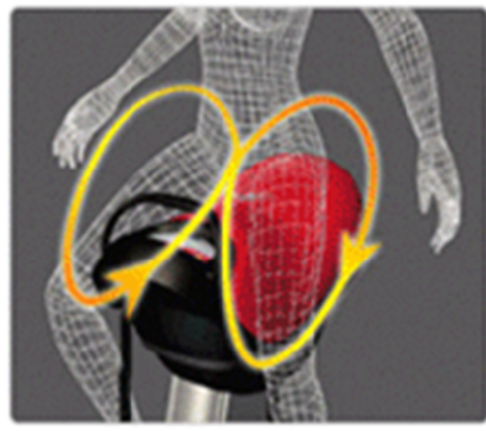

(a)

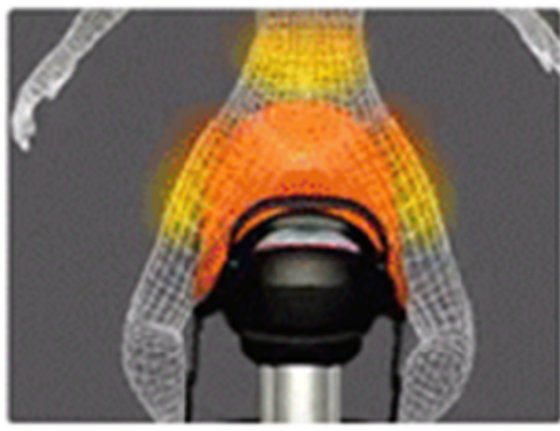

(b)

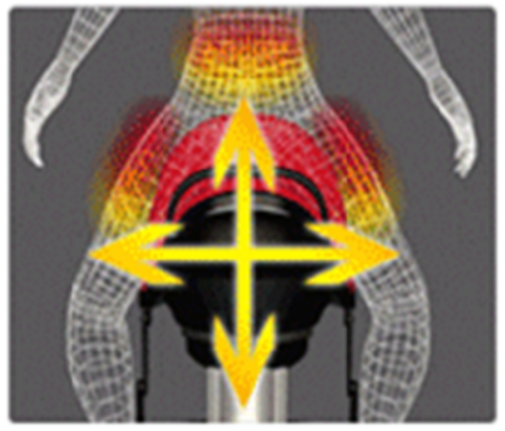

(c)

Fig. 2. (a) Movement in Postural Balance Exercise mode; (b) Movement in Abdomen Exercise mode; (c) Movement in Whole body Exercise mode.

\subsection{Horse riding exercise modes}

SRider have five exercise modes which consisted of Preparing Exercise mode, Postural Balance Exercise mode, Abdomen Exercise mode, Whole body Exercise mode and Multiple Exercise mode. SRider provides movement of extensively shaking total-body in every direction and movement of rotating based on abdomen at the same time. Figure 2 demonstrates the movement directions during the horse riding exercise in each mode. It has the different exercise velocity and exercise direction with a variety of movements as shown in Table 1. 
Table 1

Exercise velocity and exercise direction with a variety of movements in horse riding machine as SRider

\begin{tabular}{l|l|l}
\hline \hline Exercise mode & Exercise velocity (times/s) & Exercise direction \\
\hline Preparing Exercise mode & 1 times/s & left, right \\
\hline Postural Balance Exercise mode & 1.5 times/s & left, right, front, back \\
\hline Abdomen Exercise mode & 3 times/s & left, right, front, back, up, down \\
\hline Whole body Exercise mode & 3.5 times/s & left, right, front, back, up, down \\
\hline Multiple Exercise mode & Rotation in $1,1.5,3,3.5$ time/s & left, right, front, back, up, down \\
\hline
\end{tabular}

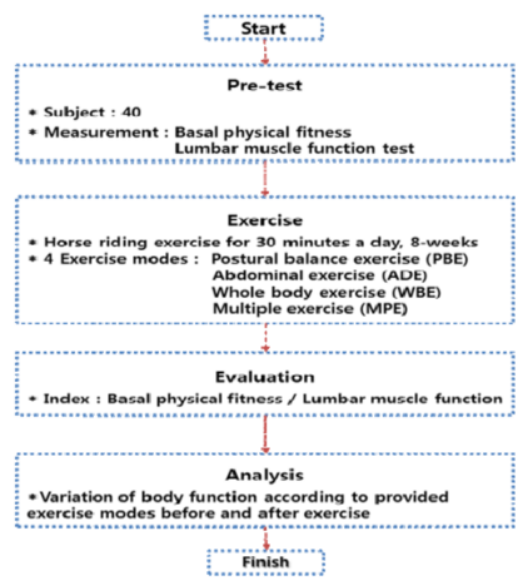

(a)

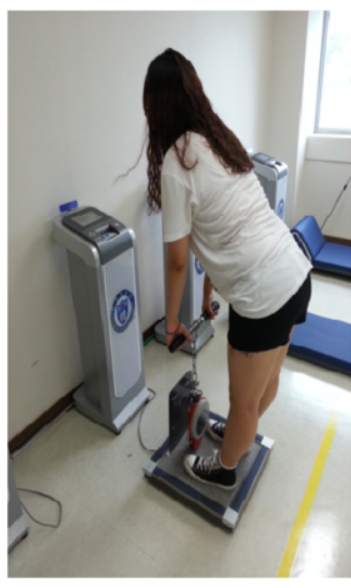

(b)

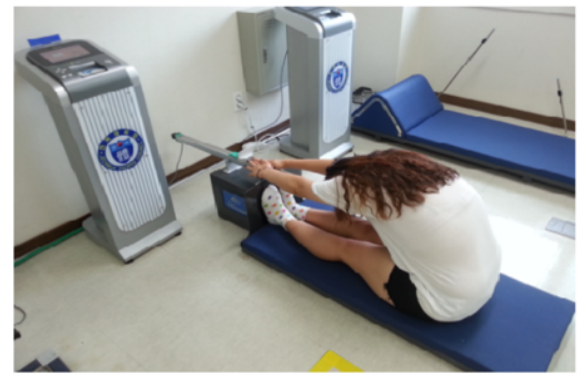

(c)

Fig. 3. (a) Horse riding training program; (b) Basal physical fitness as back muscle strength; (c) Basal physical fitness as flexibility.

\subsection{Horse riding training program}

Subjects were divided into four groups and different horse riding programs were assigned to each group using the SRider(Rider Co. \& Chonbuk National Univ, Korea). Subjects attempted to maintain their posture by stretching their waist at $90^{\circ}$, and gripped the handle softly. The legs were placed on the pedals at a right angle with eyes forward. The experiments proceeded for thirty minutes per day, three days per week, over a period of eight weeks, on the horse riding equipment [4]. Figure 3(a) is a block diagram showing the effect of the horse riding exercise on the human body. Isokinetic muscular function test was performed before and after the horse riding exercise to assess the effect of horse riding as a basal physical exercise and lumbar muscular function every four week. Indoor temperature was maintained at $21^{\circ} \mathrm{C}$ for reducing error of experimental environment.

\subsection{Evaluation of basal physical fitness}

In this study, a basal physical fitness test was performed to measure the basal physical change after the exercise. The following tests were performed: grip power, back muscle strength, isokinetic strength, sit-up, forward flexion of trunk, whole body reaction time, standing high jump, evaluation of aerobic capacity, side step, and flexibility. The back muscle strength and flexibility were selected to be 
compared with lumbar muscle function assessment for the analysis. Figures 3(b) and 3(c) show the pictures demonstrating the basal physical exercise.

\subsection{Measurement and evaluation of Isokinetic muscular function}

In this study, muscular function evaluation was performed using BIODEX system 3 , an isokinetic evaluation device shown in Figure 4(a). The evaluation was done two times before and after the exercise on horse riding equipment under different training modes.

Lumbar joint was selected and measured to verify the exercise effect, and the applied protocols were extension and flexion. When the joint torque of the subject was measured, same protocol, angular velocity, and the measurement range were provided to all groups in 4 different training modes. The general level of angular velocity used were $30 \sim 60^{\circ} / \mathrm{sec}$ for joint measurement and $90 \sim 120^{\circ} / \mathrm{sec}$ for muscle endurance [15]. Since the purpose of this research is to assess the enhancement of muscle strength, angular velocity was set between $30 \sim 60 \%$ sec for the measurement. 'Lumber torque' signifies the measurement of target muscle, elector spinae. The angular velocity was set to $60^{\circ} / \mathrm{sec}$ to observe the muscle strengthening effect, and measurement range was set to $60^{\circ}$ in overall; also, $30^{\circ}$ was set for each measurement before and after exercise while vertical position was configured as $0^{\circ}$. For lumbar joint measurement, induction of outside muscle power was blocked by fixing higher and lower limbs while keeping semi standing position to eliminate measurement error caused by ground reaction forces. Figures 4(b) and 3(c) show the measurements of lumbar torque using BIODEX system3.

The parameters used for analysis after measurement were peak torque, total work, average power, and muscle acceleration time. First, peak torque is indicated by the highest position in torque curve showing absolute maximum muscle power of a particular group of muscles. Second, total work was measured by 5 times of extension and flexion, according to the protocol provided by Biodex. The total muscle power used during 5 times of repetitive exercises is measured. The average power is a value obtained by dividing total work with real contraction time, which is used to provide ratio defining the accurate measurement of work. Finally, the muscle acceleration time is used to evaluate the agility of muscle, and it is defined as total work in $1 / 8$ second after the occurrence of muscle power. It is also defined as time spent from the starting point of torque to velocity threshold, which is the sum of angles used during the 5 times of extension/flexion exercises. Velocity threshold is obtained by the following formula:

$$
V_{t h}=\left(V_{d} \times I W_{t h}\right) / 100
$$

The following are the values used in this formula: $V_{t h}$ is the velocity threshold; $V_{d}$ is dynamometer speed; $60 \% \mathrm{sec}$ is angular velocity used for this experiment; and $I W_{t h}$ is isokinetic window threshold which was set to basic setting of $70 \%$. The obtained velocity threshold was $60 \% \mathrm{sec}$ from the calculation of $60 \mathrm{x} 0.7$. Therefore, this is the estimated time spent when the muscle is extended from $0^{\circ}$ to $42^{\circ}$, on the basis of the sum of angles accumulated during 5 times of lumbar exercises. Acceleration time $T_{a}$ is the time spent to get the maximum torque by accelerating the power of lumbar joint, and it is interpreted as reaction time of muscle related to extension/flexion exercises. 


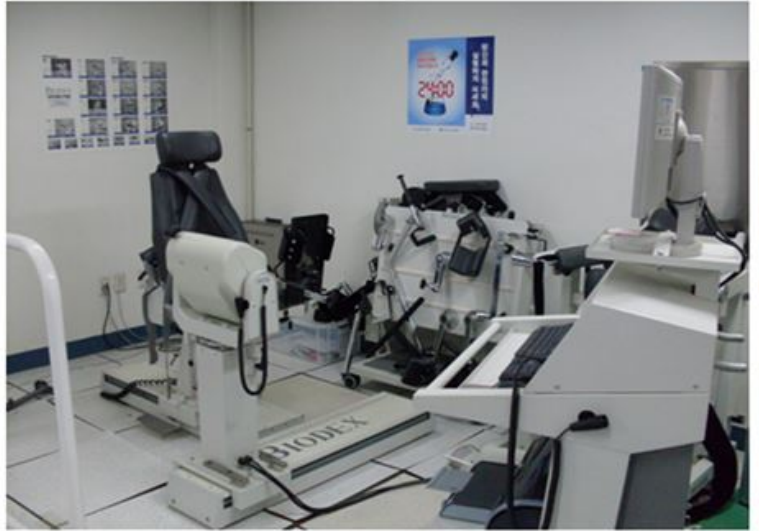

(a)

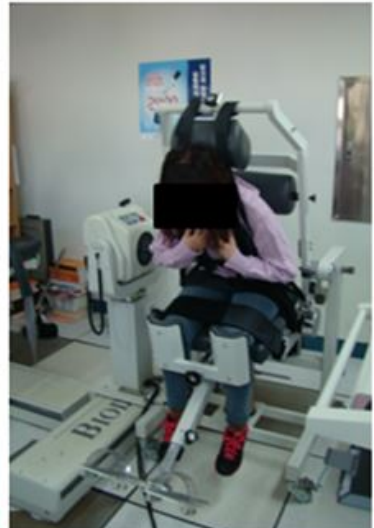

(b)

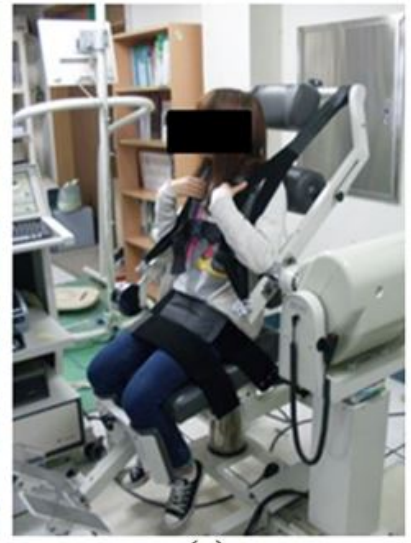

(c)

Fig. 4. (a) BIODEX system 3 as evaluation equipment of isokinetic muscular function (BIODEX Corp., NY, USA); (b) Measurement of lumbar torque during flexion using BIODEX system 3 and (c) Measurement of lumbar torque during extension using BIODEX system 3.

\subsection{Data analysis}

SPSS 18.0 for Windows (SPSS Inc., Chicago, IL, USA) was used to statistically verify the effect of horse riding exercise on lumbar torque and lumbar muscle strength; the mean and standard deviation of peak torque, total work, average power, and acceleration time were obtained by the calculation. Data are presented as mean \pm S.D. The significance of differences in the parameters of the back muscle strength, flexibility and joint torque according to training modes before and after horse riding exercise, was estimated using repeated measures analysis of variance with post hoc Bonferroni-adjusted paired $\mathrm{t}$-tests. A p-value of $<0.05$ was accepted as representing a significant difference.

\section{Results and discussion}

This study was conducted to verify the effect of indoor horse riding on basal physical exercise and lumbar muscle function. The experiment was performed with general horse riding equipment, and the changes of basal physical exercise and lumbar muscle function were analyzed by evaluating the improvements in back muscle strength, flexibility, and lumbar muscle strength, focusing on the exercise effectiveness for females.

\subsection{Basal physical exercise's change according to horse riding exercise}

The back muscle strength and flexibility were measured to observe the changes in basal physical exercise, according to different training modes of horse riding exercise. The following data was obtained after the measurement. First, back muscle strength result showed increases in PBE $(19.4 \%)$, ADE (24.4\%), WBE (35\%), and MTE (3.45\%). Back muscle strength result showed the highest effect on WBE. Flexibility result showed increase only in WBE (5.4\%), and reductions in PBE (19.6\%), $\operatorname{ADE}(1.6 \%)$, and MTE $(5.4 \%)$ in Figure 5.

The back muscle strength tended to increase the most; and this is inter-related with the lumbar torque. Back muscle strength appears to form as a combination of multiple muscles including rectus ab 


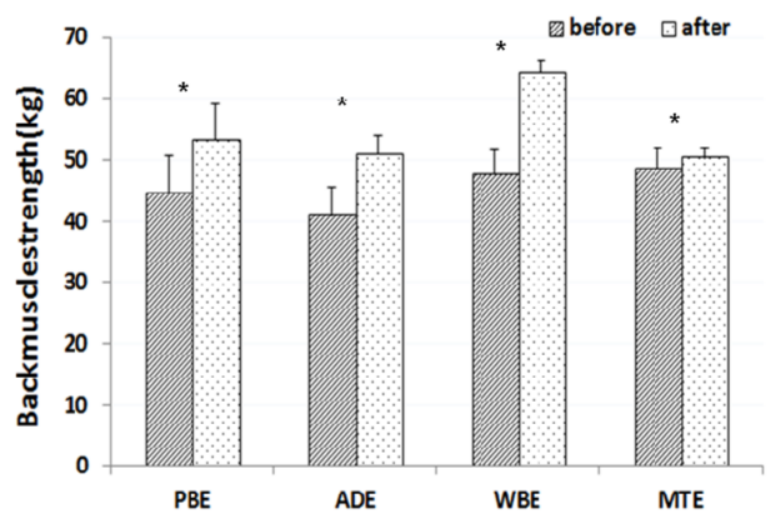

(a)

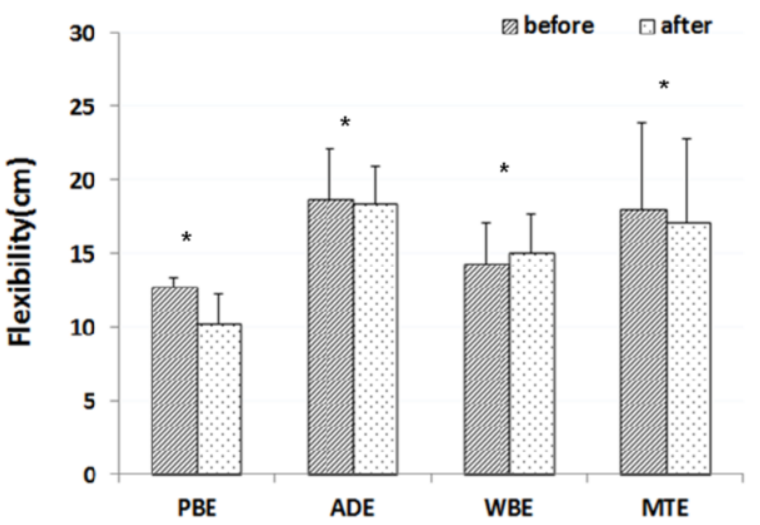

(b)

Fig. 5. Basal physical fitness before and after exercise: (a) Variation in back muscle strength and (b) Variation in flexibility.

dominis, musculus iliacus, and psoas muscle; and the strength increased as lumbar torque increased. The conclusion suggests that the horse riding is an antagonistic exercise mainly using waist and abdomen areas, and the overall effect is increased with varying degrees according to the different training modes. This also showed inter-relationship with body composition, and it showed the highest increase in whole body exercise. This explains that quick movements and wide motion range induce persistence in muscle tonus and antagonistic muscle contraction.

However, the flexibility test didn't show any significant increase regardless of mode and time of the training. This suggests that horse riding exercise has more benefits on muscle strengthening than on flexibility.

\subsection{The maximum joint torque and total work change in lumbar muscle function according to horse riding exercise}

The maximum joint torque and total work were measured and analyzed to evaluate the improving effect of muscle strength and muscle endurance of trunk, according to the training modes of horse riding exercise. The result of maximum joint torque test before the experiment were shown as follows: Postural Balance Exercise mode (Extensor, 120.95 $\pm 11.44 \mathrm{Nm}$; Flexor, $88.20 \pm 9.85 \mathrm{Nm}$ ), Abdomen Exercise mode (Extensor, $123.95 \pm 13.83 \mathrm{Nm}$; Flexor, $93.35 \pm 18.51 \mathrm{Nm}$ ), Whole body Exercise mode (Extensor, $124.23 \pm 8.98 \mathrm{Nm}$; Flexor, $91.43 \pm 8.10 \mathrm{Nm}$ ), and Multiple Exercise mode (Extensor, 123.95 \pm $13.83 \mathrm{Nm}$; Flexor, $95.90 \pm 9.10 \mathrm{Nm}$ ) in Figure 6.

Eight weeks after the experiment, the results appeared as follows: postural balance exercise mode had $153.83 \pm 13.83 \mathrm{Nm}$ (extensor increase by $27 \%$ ) and $100.93 \pm 9.85 \mathrm{Nm}$ (flexor increase by $14 \%$ ); abdomen exercise mode had $175.70 \pm 11.44 \mathrm{Nm}$ (extensor increase by $41 \%$ ) and $110.37 \pm 18.51 \mathrm{Nm}$ (flexor increase by $18 \%$ ); whole body exercise mode had $172.86 \pm 5.87 \mathrm{Nm}$ (extensor increase by $39 \%$ ) and $91.43 \pm 8.10 \mathrm{Nm}$ (flexor increase by $23 \%$ ); and multiple exercise mode had $159.92 \pm 18.09 \mathrm{Nm}$ (extensor increase by $18.6 \%$ ) and $110.20 \pm 9.50 \mathrm{Nm}$ (flexor increase by $12 \%$ ).

For the total work, there were increases in PBE (extensor 34\%, flexor 24\%), ADE (extensor 28.2\%, flexor $40 \%$ ), WBE (extensor 30.3\%, flexor 40\%), and MTE (extensor 23.4\%, flexor 17\%). The extensor showed highest increase in PBE, and WBE showed highest increase for flexor. The overall results showed the highest increase in WBE, which is similar to the maximum joint torque in Figure 7. 


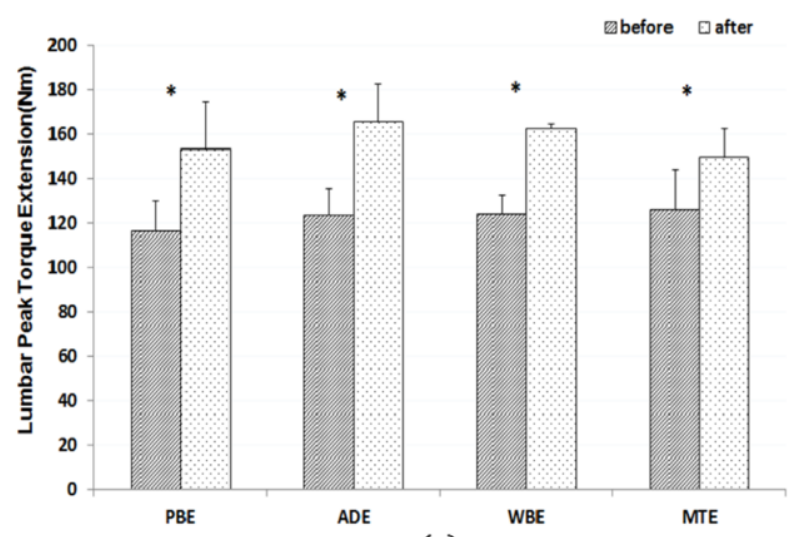

(a)

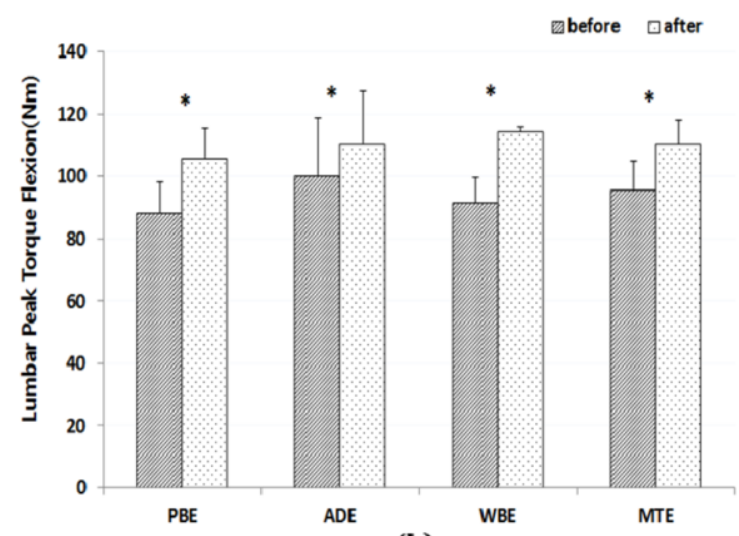

(b)

Fig. 6. Lumbar muscle functions before and after the exercise: (a) Variation in lumbar peak torque extension (Mean $\pm \mathrm{SD}$, ${ }^{*} \mathrm{p}<0.05$ ) and (b) Variation in lumbar peak torque flexion (Mean $\pm \mathrm{SD},{ }^{*} \mathrm{p}<0.05$ ).

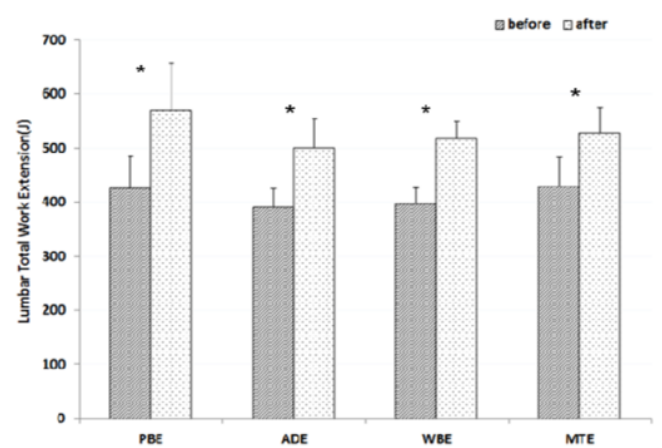

(a)

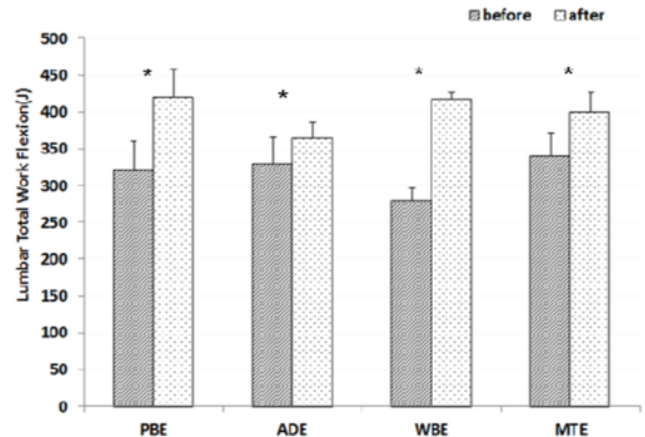

(b)

Fig. 7. Lumbar muscle functions before and after exercise: (a) Variation in lumbar total work extension (Mean $\pm \mathrm{SD},{ }^{*} \mathrm{p}<0.05$ ) (b) Variation in lumbar total work flexion (Mean $\left.\pm \mathrm{SD},{ }^{*} \mathrm{p}<0.05\right)$.

For the results of the maximum joint torque and changes in total work, lumbar torque and work were increased in all modes. The degree of increase was in the following order from highest to lowest: whole body exercise mode, abdomen exercise mode, multiple exercise mode, and postural balance exercise mode. This had similar tendency as the result of back muscle strength test. The antagonistic exercise effect resulting from rapid movements and wide motion range was the most effective in whole body exercise mode. The rapid movements provided continuous stimulations on muscle receptors to strengthen fast muscle fibers, and wide motion range provided muscle contraction stimulation to strengthen large area of antagonist.

Also, both flexor and extensor were enhanced which means the exercise had effect of strengthening agonist as well as antagonist, simultaneously. The result showed similar tendency as described above. It can be reasonably assumed that the effect was produced by circular movements in the shape of a number 8 during the horse riding exercise, which strengthens agonist and antagonist. 


\subsection{Lumbar joint average power and muscle acceleration time change in lumbar muscle function according to horse riding exercise}

Lumbar joint's average power and muscle acceleration time were measured and analyzed to observe trunks' inotropic response according to different training modes of the horse riding exercise. For average power, the result showed increases in PBE (extensor 25\%, flexor 31\%), ADE (extensor 51\%, flexor $35 \%$ ), WBE (extensor $47 \%$, flexor $46 \%$ ), and MTE (extensor $28 \%$, flexor $24 \%$ ) in Figure 8 . The extensor increased in PBE by $41.3 \%$ at the highest, and flexor increased in WBE by $47.7 \%$ at the highest. Muscle acceleration time test result showed decreases in ADE (extensor 12\%, flexor 27\%), WBE (extensor 35\%, flexor 23\%), and MTE (extensor $45 \%$, flexor $14 \%$ ). In PBE, acceleration time of flexor was reduced by $20 \%$, but that of the extensor was increased by $27.3 \%$ in Figure 9 .

Horse riding exercise is considered to reduce muscle acceleration time of lumbar joint by improving muscle contraction/relaxation response to sensory organs with continuous stimulation on nerves in muscle fibers [4]. The result in this study shows to be different from the other results in the following ways. Time was reduced the most not in whole body exercise mode but in multiple exercise mode; and rapid muscle contraction had positive effect. The rapid movements of horse riding exercise continuously stimulate the trunk muscles and improve muscle response velocity by inducing muscle fiber activation. If it is reformulated, antagonist on large area stimulates afferent fibers; and it induces active reaction to muscle extension in joint motion range to enhance the muscle strength. But this explanation will be applicable to extensor, because flexor is increased the most in abdomen exercise mode. In postural balance mode, the effect of inotropic response time decreased as muscle acceleration time increased; and this might be due to the muscle relaxation caused by slow movements and narrow motion range.

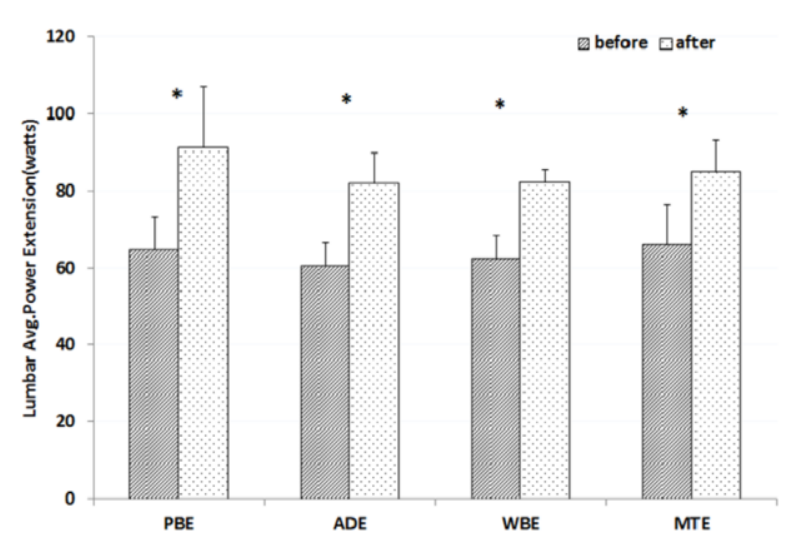

(a)

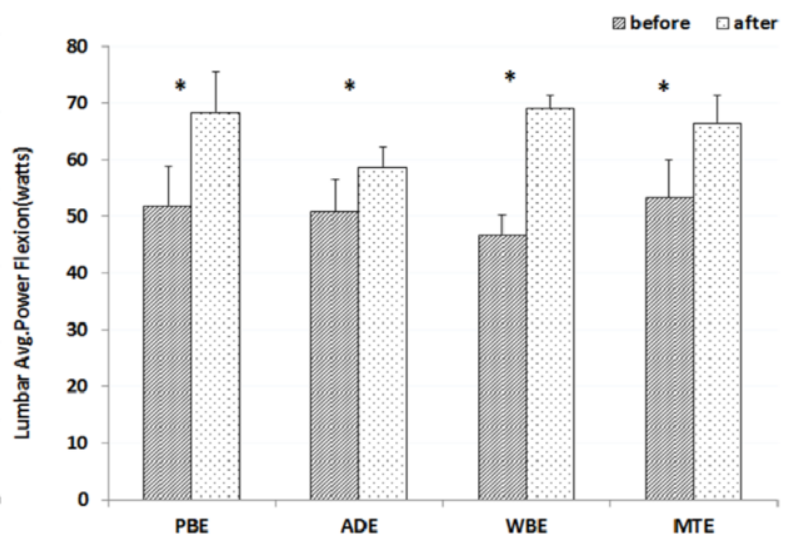

(b)

Fig. 8. Lumbar muscle functions before and after exercise: (a) Variation in lumbar average power extension (Mean $\pm \mathrm{SD}$, ${ }^{*} \mathrm{p}<0.05$ ) and (b) Variation in lumbar average power flexion (Mean $\pm \mathrm{SD},{ }^{*} \mathrm{p}<0.05$ ). 


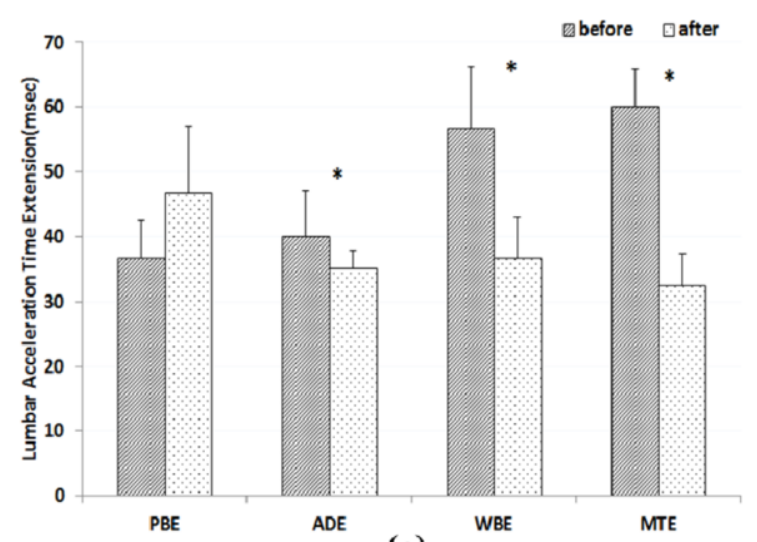

(a)

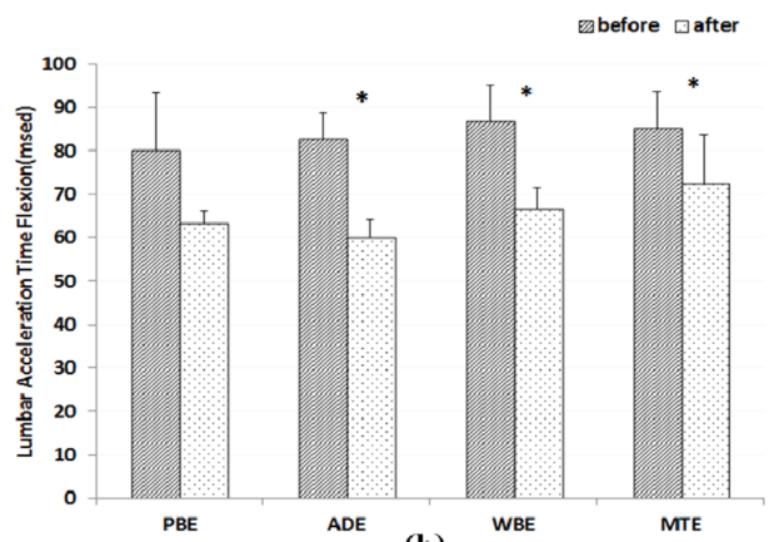

(b)

Fig. 9. Lumbar muscle functions before and after exercise: (a) Variation in lumbar acceleration time extension (Mean $\pm \mathrm{SD}$, ${ }^{*} \mathrm{p}<0.05$ ) (b) Variation in lumbar acceleration time flexion (Mean $\pm \mathrm{SD},{ }^{*} \mathrm{p}<0.05$ ).

\section{Conclusion}

This study was conducted to verify the effect of indoor horse riding on basal physical exercise and lumbar muscle function of female subjects. The indoor horse riding exercise is an effective and sufficient workout for people with limited time and capability for outdoor activities. The conclusions obtained from this research are described as follows.

Back muscle strength and lumbar joint torque test showed increases in all modes with varying degrees according to the training modes. This signifies horse riding exercise as an antagonistic exercise which is mainly focused on waist and abdomen area. The fact that both flexor and extensor increased indicates that this exercise strengthens agonist as well as antagonist. Also, the rapid movements and wide motion range induced by this exercise produce continuous muscle tonus which provides stimulations for inotropic response.

Contrary to the past reports that horse riding effects on flexibility, this study didn't show significant increase in flexibility. This suggests that indoor horse riding exercise has more benefits in strengthening muscle than in improving flexibility.

Indoor horse riding exercise is suitable with positive exercise effects for seniors or disabled persons who need muscle exercise but have difficulty in performing outdoor activities.

\section{Acknowledgment}

This work was supported by Industrial-Complex Cluster Program of the KICOX (Korea Industrial Complex Corp.) grant funded by the Korea government Ministry of Trade, Industry and Energy (1415130494) and partially supported by the Sports Promotion Fund of Seoul Olympic Sports Promotion Foundation from Ministry of Culture, Sports and Tourism. 


\section{References}

[1] C.W. Kim, Y.B. Baek and Y.S. Kwak, The effects of different types of treatment on EPOC and anti-oxidant systems after horseback riding exercise, Journal of Life Science 21 (2011), 1612-1618.

[2] S.K. Lee and J.H. Jeong, The effects of indoor horseback-riding exercise on health-related fitness, serum lipids, and defecation satisfaction of female collegiate students, Korea Sport Research 16 (2005), 153-160.

[3] M. Kubuta, M. Magasaki, M. Tokudome, Y. Shinomiya, T. Ozawa and Y. Sato, Mechanical horseback riding improves insulin sensitivity in elder diabetic patients, Diabetes Research and Clinical Practice 71 (2006), 124-130.

[4] S.R. Kang, C.H. Yu, G.Y. Jung, D.A. Moon, S.Y. Park, J.J. Kim and T.K. Kwon, Analysis of the indoor horse riding exercise equipment on the young people, International Journal of Precision Engineering and Manufacturing 14 (2013), $1471-1478$.

[5] Y. Mitani, K. Doi, T. Yano, E. Sakamaki, K. Mukai, Y. Shinomiya and T. Kimura, Effect of exercise using a horseriding simulator on physical ability of frail seniors, Journal of Physical Therapy Science 20 (2008), 177-183.

[6] Y.P. Wuang, C.C. Wang, M.H. H and C.Y. Su, The effectiveness of simulated developmental horse-riding program in children with autism, Adapted Physical Activity Quarterly 27 (2010), 113-126.

[7] M.B.S. Borges, M.J.S. Werneck, M.L. Silva, L. Gandolfi and R. Pratesi, Therapeutic effects of a horse riding simulator in children with cerebral palsy, Files of Neuro-Psychiatry 69 (2011), 799-804.

[8] J.Y. Han, J.M. Kim, S.K. Kim, J.S. Chung, H.C. Lee, J.K. Lim, J.W. Lee and K.Y. Park, Therapeutic effects of mechanical horseback riding on gait and balance ability, Annals Rehabilitation Medicine 26 (2012), 762-769.

[9] D.B. Bertoti, Effect of therputic horseback riding on posture in children with cerebral palsy, Journal of Physical Therapy 68 (1998), 1505-1512.

[10] W.Y. Oh, C.C. Ryew, J.H. Kim and S.H. Hyun, Kinematic analysis of horse-riding posture according to skill levels during rising trot with JeJu-horse, Korean Journal of Sport Biomechanics 19 (2009), 467-479.

[11] S.C. Han, H.K. Choo and S.H. Lee, The effects of horseback riding on the balance improvement of the children with cerebral palsy, The Korean Journal of Physical Education 43 (2004), 601-610.

[12] H. Yoshiyuki, N. Masaru, B. Gustavo, S. Youichi, O. Takahisa and S. Yuzo, Effects of daily mechanical horseback riding on insulin sensitivity and resting metabolism in middle-aged type 2 diabetes mellitus patients, Nagoya Journal of Medical Science 72 (2010), 129-137.

[13] H.A. Alfredson, G. Hedberg, E. Bergstrome, P. Nordstrom and R. Lorentzon, High thigh muscle strength but not bone mass in young horseback-riding female, Calcified Tissue International 62 (1998), 497-501.

[14] M.F. Devienne and C.Y. Guezennee, Energy expenditure of horse riding, European Journal of Applied Physiology 82 (2000), 499-503.

[15] S.R. Kang, S.B. Seo, G.Y. Jeong, J.J Bae, C.H. Yu, M. Yu, D.A. Moon, J.S. Jeong and T.K. Kwon, Effect on improvement of muscle strength imbalance according to load deviation pattern of left and right arms in upper limbs, Journal of the Korean Society for Precision Engineering 29 (2012), 1026-1034. 